\title{
A Survey on the Classical Limit of Quantum Dynamical Entropies
}

\author{
V. CAPpellini \\ "Mark Kac" Complex Systems Research Centre, Uniwersytet Jagielloński \\ Reymonta 4, 30-059 Kraków, Poland \\ and \\ Center for Theoretical Physics, Polish Academy of Sciences \\ al. Lotników 32/46, 02-668 Warszawa, Poland

\begin{abstract}
We analyze the behavior of quantum dynamical entropies production from sequences of quantum approximants approaching their (chaotic) classical limit. The model of the quantized hyperbolic automorphisms of the 2-torus is examined in detail and a semi-classical analysis is performed on it using coherent states, fulfilling an appropriate dynamical localization property. Correspondence between quantum dynamical entropies and the Kolmogorov-Sinai invariant is found only over timescales that are logarithmic in the quantization parameter.
\end{abstract}

PACS numbers: 05.45.-a, 05.45.Mt, 05.45.Ac, 03.65.Fd

\section{Introduction}

The notion of classical chaos is associated with motion on a compact phase-space with high sensitivity to initial conditions: trajectories diverge exponentially fast and nevertheless remain confined to bounded regions [1-7].

In an opposite way, quantization on compacts yields discrete energy spectra, which entail quasi-periodic time-evolution [8].

Nevertheless, nature is fundamentally quantal and, according to the correspondence principle, classical behavior must emerge in the limit $\hbar \rightarrow 0$.

Also, classical and quantum mechanics are expected to overlap over times expected to scale as $\hbar^{-\alpha}$ for some $\alpha>0$ [7], the so-called semi-classical regime. Actually, it turns out that this is true only for regular classical limits whereas, for chaotic ones, classical and quantum mechanics agree over times which scale as $-\log \hbar[5-7]$, and footprints of the exponential separation of classical trajectories are found even on finite dimensional quantization provided that the time does not exceed such a logarithmic upper bound $[6,9]$. Both timescales diverge when $\hbar \rightarrow 0$, but the shortness of the latter means that classical mechanics has to be replaced by quantum mechanics much sooner for quantum systems with chaotic 
classical behavior. The logarithmic breaking time $-\log \hbar$ has been considered by some as a violation of the correspondence principle $[10,11]$ and by others, see [6] and Chirikov in [5], as the evidence that time and classical limits do not commute.

The analytic studies of logarithmic timescales have been mainly performed by means of semi-classical tools, essentially by focusing, via coherent state techniques, on the phase space localization of specific time evolving quantum observables. In the following, we shall show how they emerge in the context of quantum dynamical entropies.

As a particular example, we shall concentrate on finite dimensional quantizations of continuous hyperbolic automorphisms of the 2-torus $\mathbb{T}^{2}:=\mathbb{R}^{2} / \mathbb{Z}^{2}$ (the unit square with opposite sides identified), which are prototypes of chaotic behavior; indeed, their trajectories separate exponentially fast with a Lyapunov exponent $\log \lambda_{+}>0[12,13]$. If $\delta$ is an initial error along a trajectory, and $\delta_{n} \simeq \delta \lambda_{+}^{n}$ its classical spreading after $n$ steps of the (time-stroboscopic) dynamics, then boundness of the motion imposes $\delta_{n} \leq 1$, where 1 is the diameter of the 2-torus $\mathbb{T}^{2}$. This explains why the limit $\delta \rightarrow 0$ has necessarily to be performed before the time-limit, and the Lyapunov exponent can be computed as

$$
\log \lambda_{+}=\lim _{n \rightarrow \infty} \frac{1}{n} \lim _{\delta \rightarrow 0} \log \left(\frac{\delta_{n}}{\delta}\right) .
$$

Standard quantization, à la Berry, of hyperbolic automorphisms on $\mathbb{T}^{2}[14,15]$ yields Hilbert spaces of a finite dimension $N$, this latter variable playing the role of the semi-classical parameter and setting to $1 / N$ the minimal size of the phase-space grain cells. Imposing the latter bound, $\min \{\delta\} \geq 1 / N$, it is evident how the conflict between the two limits, emerging once $\delta_{n} \approx 1$, can be transferred in the time-step $n$ as $n \simeq \log N / \log \lambda_{+}$. In this sense, rather than a violation of the correspondence principle, the logarithmic breaking-time indicates the typical scaling for a joint time-classical limit suited to classically chaotic quantum systems.

The Kolmogorov-Sinai dynamical entropy [3] (KS-entropy, for short) is defined by assigning measures to bunches of trajectories and computing the Shannon-entropy per time-step of the ensemble of bunches in the limit of infinitely many time-steps: The more chaotic the time-evolution, the more the possible bunches and the larger their entropy. The production of different bunches of trajectories issuing from the same bunch is typical of high sensitivity to initial conditions and this is indeed the mechanism at the basis of the theorem of Ruelle and Pesin [16], linking KS-entropy of a smooth, classical dynamical systems, to the sum of its positive Lyapunov exponents.

In the quantum realm, there are different candidates for non-commutative extensions of the KS-invariant [17-21]: in this paper we shall focus on one of them, called ALF-entropy [18], and we shall study its semi-classical limit.

The ALF-entropy is based on the algebraic properties of dynamical systems, that is on the fact that they are describable by suitable algebras of observables, 
their time evolution by linear maps on these algebras, and their states by expectations over them.

We show that, while being bounded by $\log N$, nevertheless over numbers of time steps $1 \ll n<\log N$, the entropy content per letter, or entropy production, is $\log \lambda_{+}$. It thus follows that the joint limit $n, N \rightarrow+\infty$, with $n \propto \log N$, yields the Kolmogorov-Sinai entropy. This confirms the numerical results in [22, 23], where the dynamical entropy [18] is applied to the study of the quantum kicked top, respectively to quantum cat maps.

In this approach, the presence of logarithmic timescales indicates the typical scaling for a joint time/classical limit suited to preserve positive entropy production in quantized classically chaotic quantum systems.

The paper is organized as follows: Sect. 2 contains a brief review of the algebraic approach to classical and dynamical systems, while Sect. 3 introduces some basic semi-classical tools. Sections 4 and 5 deal with the quantization of hyperbolic maps on finite dimensional Hilbert spaces and the relation between classical and time limits. Section 6 gives an overview of various models of quantum dynamical entropies present in the literature and particularly focus on the one proposed by Alicki and Fannes [18, 24] (ALF-entropy, where L stands for Lindblad). Finally, in Sect. 7, the semi-classical behavior of quantum dynamical entropies is studied and the emergence of a typical logarithmic timescale is showed.

\section{Dynamical systems: algebraic setting}

Usually, continuous classical motion is described by means of a measure space $\mathcal{X}$, the phase-space, endowed with the Borel $\sigma$-algebra and a normalized measure $\mu, \mu(\mathcal{X})=1$. The "volumes"

$$
\mu(E)=\int_{E} \mu(\mathrm{d} \boldsymbol{x})
$$

of measurable subsets $E \subseteq \mathcal{X}$ represent the probabilities that phase-points $\boldsymbol{x} \in$ $\mathcal{X}$ belong to them. By specifying the statistical properties of the system, the measure $\mu$ defines a "state" of it. In such a scheme, a reversible discrete time dynamics amounts to an invertible measurable map $T$ onto $\mathcal{X}$ such that $\mu \circ T=\mu$, and to its iterates $\left\{T^{k} \mid k \in \mathbb{Z}\right\}: T$-invariance of the measure $\mu$ ensures that the state defined by $\mu$ can be taken as an equilibrium state with respect to the given dynamics. Phase-trajectories passing through $\boldsymbol{x} \in \mathcal{X}$ at time 0 are then sequences $\left\{T^{k} \boldsymbol{x} \mid k \in \mathbb{Z}\right\}[3]$. Classical dynamical systems are thus conveniently described by triplets $(\mathcal{X}, T, \mu)$. In the present work we shall focus upon the following:

- $\mathcal{X}$ - a compact metric space: the 2-dimensional torus $\mathrm{T}^{2}=\mathbb{R}^{2} / \mathbb{Z}^{2}=$ $\left\{\left(x_{1}, x_{2}\right) \in \mathbb{R}^{2} \quad(\bmod 1)\right\} ;$

- $T$ - invertible measurable transformations from $\mathcal{X}$ to itself such that $T^{-1}$ are also measurable;

- $\mu$ - the Lebesgue measure $\mu(\mathrm{d} \boldsymbol{x})=\mathrm{d} x_{1} \mathrm{~d} x_{2}$ on $\mathbb{T}^{2}$. 
In this paper, we consider a general scheme for quantizing and dequantizing, i.e. for taking the classical limit (see [25]). Within this framework, we focus on the semi-classical limit of quantum dynamical entropies of finite dimensional quantizations of the celebrated Arnold cat map and of generic maps belonging to the so-called unimodular group on the 2-torus: in the following we simply denote such a family of maps cat maps family. The last denomination is perfectly legitimate, in fact the acronym CAT stands for continuous automorphism of the torus.

In order to make the quantization procedure more explicit, it proves useful to follow an algebraic approach and replace $(\mathcal{X}, T, \mu)$ with $\left(\mathfrak{M}_{\mu}, \Theta, \omega_{\mu}\right)$ where:

- $\mathfrak{M}_{\mu}$ is the von Neumann algebra $\mathfrak{L}_{\mu}^{\infty}(\mathcal{X})$ of (equivalence classes of) essentially bounded $\mu$-measurable functions on $\mathcal{X}$, equipped with the so-called essential supremum norm $\|\cdot\|_{\infty}[26]$;

- $\left\{\Theta^{k} \mid k \in \mathbb{Z}\right\}$ is the discrete group of automorphisms of $\mathfrak{M}_{\mu}$ which implements the dynamics: $\Theta(f):=f \circ T^{-1}$. The invariance of the reference measure reads now $\omega_{\mu} \circ \Theta=\omega_{\mu}$;

- $\omega_{\mu}$ is the state on $\mathfrak{M}_{\mu}$ defined by the reference measure $\mu$ :

$$
\omega_{\mu}: \mathfrak{M}_{\mu} \ni f \longmapsto \omega_{\mu}(f):=\int_{\mathcal{X}} \mu(\mathrm{d} \boldsymbol{x}) f(x) \in \mathbb{R}^{+} .
$$

Quantum dynamical systems are described in a completely similar way by a triple $(\mathfrak{M}, \Theta, \omega)$, the critical difference being that the algebra of observables $\mathfrak{M}$ is no longer Abelian:

- $\mathfrak{M}$ is a von Neumann algebra of operators, the observables, acting on a Hilbert space $\mathfrak{H}$;

- $\Theta$ is an automorphism of $\mathfrak{M}$;

- $\omega$ is an invariant normal state on $\mathfrak{M}: \omega \circ \Theta=\omega$.

Quantizing essentially corresponds to suitably mapping the commutative, classical triple $\left(\mathfrak{M}_{\mu}, \Theta, \omega_{\mu}\right)$ to a non-commutative, quantum triple $(\mathfrak{M}, \Theta, \omega)$.

\section{Classical limit: coherent states}

Performing the classical limit or a semi-classical analysis consists in studying how a family of algebraic triples $(\mathfrak{M}, \Theta, \omega)$, depending on a quantization $\hbar$-like parameter, is mapped onto $\left(\mathfrak{M}_{\mu}, \Theta, \omega_{\mu}\right)$ when the parameter goes to zero. The most successful semi-classical tools are based on the use of coherent states (CS for short).

For our purposes, we shall use a large integer $N$ as a quantization parameter, i.e. we use $1 / N$ as the $\hbar$-like parameter. In fact, we shall consider cases where $\mathfrak{M}$ is the algebra $\mathcal{M}_{N}$ of $N$-dimensional square matrices acting on $\mathbb{C}^{N}$, the quantum reference state is the normalized trace $\frac{1}{N} \operatorname{Tr}$ on $\mathcal{M}_{N}$, denoted by $\tau_{N}$, and the dynamics is given in terms of a unitary operator $U_{T}$ on $\mathbb{C}^{N}$ in the standard way: $\Theta_{N}(X):=U_{T}^{*} X U_{T}$. 
In full generality, coherent states will be identified as follows.

Definition 3.1. A family $\left\{\left|C_{N}(\boldsymbol{x})\right\rangle \mid \boldsymbol{x} \in \mathcal{X}\right\} \in \mathfrak{H}$ of vectors, indexed by points $\boldsymbol{x} \in \mathcal{X}$, constitutes a set of coherent states if it satisfies the following requirements:

1. Measurability: $\boldsymbol{x} \mapsto\left|C_{N}(\boldsymbol{x})\right\rangle$ is measurable on $\mathcal{X}$;

2. Normalization: $\left\|C_{N}(\boldsymbol{x})\right\|^{2}=1, \boldsymbol{x} \in \mathcal{X}$;

3. Overcompleteness: $N \int_{\mathcal{X}} \mu(\mathrm{d} \boldsymbol{x})\left|C_{N}(\boldsymbol{x})\right\rangle\left\langle C_{N}(\boldsymbol{x})\right|=\mathbb{1}_{N}$;

4. Localization: given $\varepsilon>0$ and $d_{0}>0$, there exists $N_{0}\left(\epsilon, d_{0}\right)$ such that for $N \geq N_{0}$ and $d_{\mathcal{X}}(\boldsymbol{x}, \boldsymbol{y}) \geq d_{0}$ one has

$$
N\left|\left\langle C_{N}(\boldsymbol{x}), C_{N}(\boldsymbol{y})\right\rangle\right|^{2} \leq \varepsilon
$$

The symbol $d_{\mathcal{X}}(\boldsymbol{x}, \boldsymbol{y})$ used in the localization property stands for the length of the shorter segment connecting the two points on $\mathcal{X}$. Of course the latter quantity does depend on the topological properties of $\mathcal{X}$. In particular, for the 2-torus,

$$
d_{\mathbb{T}^{2}} \boldsymbol{x}, \boldsymbol{y}:=\min _{\boldsymbol{n} \in \mathbb{Z}^{2}}\|\boldsymbol{x}-\boldsymbol{y}+\boldsymbol{n}\|_{\mathbb{R}^{2}} .
$$

The overcompleteness condition may be written in dual form as

$$
N \int_{\mathcal{X}} \mu(\mathrm{d} \boldsymbol{x})\left\langle C_{N}(\boldsymbol{x}), X C_{N}(\boldsymbol{x})\right\rangle=\operatorname{Tr} X, \quad X \in \mathcal{M}_{N} .
$$

Indeed,

$$
N \int_{\mathcal{X}} \mu(\mathrm{d} \boldsymbol{x})\left\langle C_{N}(\boldsymbol{x}), X C_{N}(\boldsymbol{x})\right\rangle=N \operatorname{Tr}\left(\int_{\mathcal{X}} \mu(\mathrm{d} \boldsymbol{x})\left|C_{N}(\boldsymbol{x})\right\rangle\left\langle C_{N}(\boldsymbol{x})\right| X\right)=\operatorname{Tr} X .
$$

\subsection{Anti-Wick quantization}

In order to study the classical limit and, more generally, the semi-classical behavior of $\left(\mathcal{M}_{N}, \Theta_{N}, \tau_{N}\right)$ when $N \rightarrow \infty$, we introduce two linear maps. The first, $\gamma_{N \infty}$ (anti-Wick quantization) associates $N \times N$ matrices of $\mathcal{M}_{N}$ to functions in $\mathfrak{M}_{\mu}=\mathfrak{L}_{\mu}^{\infty}(\mathcal{X})$; the second one, $\gamma_{\infty N}$, maps $N \times N$ matrices to functions in $\mathfrak{L}_{\mu}^{\infty}(\mathcal{X})$. Definition 3.2. Given a family $\left\{\left|C_{N}(\boldsymbol{x})\right\rangle \mid \boldsymbol{x} \in \mathcal{X}\right\}$ of $C S$ in $\mathbb{C}^{N}$, the anti-Wick quantization scheme will be described by a (completely) positive unital map $\gamma_{N_{\infty}}$ : $\mathfrak{M}_{\mu} \rightarrow \mathcal{M}_{N}$

$$
\mathfrak{M}_{\mu} \ni f \mapsto N \int_{\mathcal{X}} \mu(\mathrm{d} \boldsymbol{x}) f(\boldsymbol{x})\left|C_{N}(\boldsymbol{x})\right\rangle\left\langle C_{N}(\boldsymbol{x})\right|=: \gamma_{N \infty}(f) \in \mathcal{M}_{N} .
$$

The corresponding dequantizing map $\gamma_{\infty N}: \mathcal{M}_{N} \rightarrow \mathfrak{M}_{\mu}$ will correspond to the (completely) positive unital map

$$
\mathcal{M}_{N} \ni X \mapsto\left\langle C_{N}(\boldsymbol{x}), X C_{N}(\boldsymbol{x})\right\rangle=: \gamma_{\infty N}(X)(\boldsymbol{x}) \in \mathfrak{M}_{\mu} .
$$

Both maps are identity preserving because of the conditions imposed on the CS-family and are also completely positive, since the domain of $\gamma_{N \infty}$ is a commutative algebra as well as the range of $\gamma_{\infty N}$. The following two equivalent properties are less trivial: 
Proposition 3.1. For all $f \in \mathfrak{M}_{\mu}$

$$
\lim _{N \rightarrow \infty} \gamma_{\infty N} \circ \gamma_{N \infty}(f)=f, \quad \mu-\text { a.e. }
$$

Proposition 3.2. For all $f, g \in \mathfrak{M}_{\mu}$

$$
\lim _{N \rightarrow \infty} \tau_{N}\left(\gamma_{N \infty}(f)^{*} \gamma_{N \infty}(g)\right)=\omega_{\mu}(\bar{f} g)=\int_{\mathcal{X}} \mu(\mathrm{d} \boldsymbol{x}) \overline{f(\boldsymbol{x})} g(\boldsymbol{x}) .
$$

The previous two propositions, proved in [27, 28], can be taken as requests on any well-defined quantization/dequantization scheme for observables. In the sequel, we shall need the notion of quantum dynamical systems $\left(\mathcal{M}_{N}, \Theta_{N}, \tau_{N}\right)$ tending to the classical limit $\left(\mathfrak{M}_{\mu}, \Theta, \omega_{\mu}\right)$. We then not only need convergence of observables but also of the dynamics. This aspect will be considered in Sect. 5 .

\section{Classical and quantum cat maps}

In this section, we collect the basic material needed to describe both classical and quantum cat maps and we introduce a specific set of CS that will enable us to perform the semi-classical analysis over such dynamical systems.

\subsection{Finite dimensional quantizations}

We first introduce cat maps in the spirit of the algebraic formulation introduced in the previous sections.

Definition 4.1. Hyperbolic continuous automorphisms of the torus are generically represented by triples $\left(\mathfrak{M}_{\mu}, \Theta, \omega_{\mu}\right)$, where

- $\mathfrak{M}_{\mu}$ is the algebra of essentially bounded functions on the two-dimensional torus $\mathbb{T}^{2}:=\mathbb{R}^{2} / \mathbb{Z}^{2}=\left\{\left(x_{1}, x_{2}\right) \in \mathbb{R}^{2}(\bmod 1)\right\}$, equipped with the Lebesgue measure $\mu(\mathrm{d} \boldsymbol{x}):=\mathrm{d} \boldsymbol{x} ;$

- $\left\{\Theta^{k} \mid k \in \mathbb{Z}\right\}$ is the family of automorphisms (discrete time evolution) given by $\mathfrak{M}_{\mu} \ni f \mapsto\left(\Theta^{k} f\right)(\boldsymbol{x}):=f\left(A^{-k} \boldsymbol{x}(\bmod 1)\right)$, where $A=\left(\begin{array}{ll}a & b \\ c & d\end{array}\right)$ has integer entries such that $a d-b c=1,|a+d|>2$ and maps $\mathrm{T}^{2}$ onto itself;

- $\omega_{\mu}$ is the expectation obtained by integration with respect to the Lebesgue measure: $\mathfrak{M}_{\mu} \ni f \mapsto \omega_{\mu}(f):=\int_{\mathbb{T}^{2}} \mathrm{~d} \boldsymbol{x} f(\boldsymbol{x})$, that is left invariant by $\Theta$.

Denoting with $t:=\operatorname{Tr}(A) / 2$ the semi-trace of $A,|t|>1$, the two irrational eigenvalues of $A$ can be written as $1<\lambda_{+}:=t+\sqrt{t^{2}-1}$ and $1>\lambda_{-}:=$ $t-\sqrt{t^{2}-1}=\lambda_{+}^{-1}$. Distances are stretched along the direction of the eigenvector $\left|\boldsymbol{e}_{+}\right\rangle, A\left|\boldsymbol{e}_{+}\right\rangle=\lambda_{+}\left|\boldsymbol{e}_{+}\right\rangle$, contracted along that of $\left|\boldsymbol{e}_{-}\right\rangle, A\left|\boldsymbol{e}_{-}\right\rangle=\lambda_{-}\left|\boldsymbol{e}_{-}\right\rangle$and all periodic points are hyperbolic [29]. Once the folding condition is added, the hyperbolic automorphisms of the torus become prototypes of classical chaos, with positive Lyapunov exponent $\log \lambda_{+}$.

One can quantize the associated algebraic triple $\left(\mathfrak{M}_{\mu}, \Theta, \omega_{\mu}\right)$ on either infinite [30] or finite dimensional Hilbert spaces [14, 15, 31]. In the following, we shall focus on the latter. 
Given an integer $N$, we consider an orthonormal basis $|j\rangle$ of $\mathbb{C}^{N}$, where the index $j$ runs through the residual class modulo $N$, here and in the following denoted by $(\mathbb{Z} / N \mathbb{Z})$, namely $|j+N\rangle \equiv|j\rangle, j \in \mathbb{Z}$. Using this basis we define two unitary matrices $U_{N}$ and $V_{N}$, representing position and momentum shift operators, as follows:

$$
U_{N}|j\rangle:=\exp \left(\frac{2 \pi \mathrm{i}}{N} u\right)|j+1\rangle, \quad \text { and } \quad V_{N}|j\rangle:=\exp \left(\frac{2 \pi \mathrm{i}}{N}(v-j)\right)|j\rangle .
$$

In the last equation, we explicitly indicated the dependence on two arbitrary phases $(u, v) \in[0,1)$ labeling the representation and fulfilling

$$
U_{N}^{N}=\mathrm{e}^{2 \mathrm{i} \pi u} \mathbb{1}_{N}, \quad V_{N}^{N}=\mathrm{e}^{2 \mathrm{i} \pi v} \mathbb{1}_{N} .
$$

It turns out that

$$
U_{N} V_{N}=\exp \left(\frac{2 \mathrm{i} \pi}{N}\right) V_{N} U_{N}
$$

Introducing Weyl operators labeled by $\boldsymbol{n}=\left(n_{1}, n_{2}\right) \in \mathbb{Z}^{2}$

$$
W_{N}(\boldsymbol{n}):=\exp \left(\frac{\mathrm{i} \pi}{N} n_{1} n_{2}\right) V_{N}^{n_{2}} U_{N}^{n_{1}}=W_{N}(-\boldsymbol{n})^{*},
$$

it follows that

$$
\begin{aligned}
& W_{N}(N \boldsymbol{n})=\mathrm{e}^{\mathrm{i} \pi\left(N n_{1} n_{2}+2 n_{1} u+2 n_{2} v\right)} \\
& W_{N}(\boldsymbol{n}) W_{N}(\boldsymbol{m})=\exp \left(\frac{\mathrm{i} \pi}{N} \sigma(\boldsymbol{n}, \boldsymbol{m})\right) W_{N}(\boldsymbol{n}+\boldsymbol{m}),
\end{aligned}
$$

where $\sigma(\boldsymbol{n}, \boldsymbol{m}):=n_{1} m_{2}-n_{2} m_{1}$ is the so-called symplectic form.

Definition 4.2. Quantized cat maps will be identified with triples $\left(\mathcal{M}_{N}, \Theta_{N}, \tau_{N}\right)$ where

- $\mathcal{M}_{N}$ is the full $N \times N$ matrix algebra over $\mathbb{C}$ generated by the (discrete) group of Weyl operators $\left\{W_{N}(\boldsymbol{n}) \mid \boldsymbol{n} \in \mathbb{Z}^{2}\right\}$. In the following, such a group will be denoted by Weyl group;

- $\Theta_{N}: \mathcal{M}_{N} \mapsto \mathcal{M}_{N}$ is the automorphism such that

$$
W_{N}(\boldsymbol{p}) \mapsto \Theta_{N}\left(W_{N}(\boldsymbol{p})\right):=W_{N}(A \boldsymbol{p}), \quad \boldsymbol{p} \in(\mathbb{Z} / N \mathbb{Z})^{2} .
$$

In the definition of above, we have omitted reference to the parameters $u, v$ in (3): they must be chosen such that

$$
\left(\begin{array}{ll}
a & c \\
b & d
\end{array}\right)\left(\begin{array}{l}
u \\
v
\end{array}\right)=\left(\begin{array}{l}
u \\
v
\end{array}\right)+\frac{N}{2}\left(\begin{array}{l}
a c \\
b d
\end{array}\right) \quad(\bmod 1)
$$

Then, the folding condition (4) is compatible with the time evolution [15]. The reason for (9) is the following: denoting with $\hat{\boldsymbol{e}}_{1}$ and $\hat{\boldsymbol{e}}_{2}$ the standard unit vectors of $\mathbb{R}^{2}$, the representation generated by the two generators $U_{N}=W_{N}\left(\hat{\boldsymbol{e}}_{1}\right)$ and $V_{N}=W_{N}\left(\hat{\boldsymbol{e}}_{2}\right)$ and the one generated by $\Theta_{N}\left(U_{N}\right)=W_{N}\left(A \hat{\boldsymbol{e}}_{1}\right)$ and $\Theta_{N}\left(V_{N}\right)=$ $W_{N}\left(A \hat{e}_{2}\right)$ must be unitarily equivalent; in other words the two representations must be labeled by the same $u$ and $v$. According to (4), this can be expressed by

$$
\left[W_{N}\left(\hat{\boldsymbol{e}}_{1}\right)\right]^{N}=\left[W_{N}\left(A \hat{\boldsymbol{e}}_{1}\right)\right]^{N} \quad \text { and } \quad\left[W_{N}\left(\hat{\boldsymbol{e}}_{2}\right)\right]^{N}=\left[W_{N}\left(A \hat{\boldsymbol{e}}_{2}\right)\right]^{N} ;
$$

the latter equation restricts the possible couples $(u, v)$ available and leads to (9). 
An important set of matrices $A$, originally called "set of quantizable maps" and characterized by $(u, v)=(0,0)$, is also important for historical reasons, indeed it was the set used by Berry and Hannay [32] to develop the first quantization of cat maps. Recent developments of Berry's approach to quantization can be found in $[33-35]$.

Further, relation (7b) is also preserved since the condition $\operatorname{det} A=1$ guarantees that the symplectic form remains invariant, i.e. $\sigma(A \boldsymbol{n}, A \boldsymbol{m})=\sigma(\boldsymbol{n}, \boldsymbol{m})$. Invariance of $\sigma(\cdot, \cdot)$, together with $(7)$, also allows Eq. (8) to hold true for all $\boldsymbol{p} \in \mathbb{Z}^{2}$ and not only for those in $(\mathbb{Z} / N \mathbb{Z})^{2}$.

Many other useful relations can be obtained by using the explicit expression

$$
W_{N}(\boldsymbol{n})|j\rangle=\exp \left(\frac{\mathrm{i} \pi}{N}\left(-n_{1} n_{2}+2 n_{1} u+2 n_{2} v\right)\right) \exp \left(-\frac{2 \mathrm{i} \pi}{N} j n_{2}\right)\left|j+n_{1}\right\rangle .
$$

In particular, from (11) one readily derives the decomposition

$$
\mathcal{M}_{N} \ni X=\sum_{\boldsymbol{m} \in(\mathbb{Z} N \mathbb{Z})^{2}} \tau_{N}\left(X W_{N}(-\boldsymbol{m})\right) W_{N}(\boldsymbol{m}),
$$

while from Eq. (7b) one gets

$$
\left[W_{N}(\boldsymbol{n}), W_{N}(\boldsymbol{m})\right]=2 \mathrm{i} \sin \left(\frac{\pi}{N} \sigma(\boldsymbol{n}, \boldsymbol{m})\right) W_{N}(\boldsymbol{n}+\boldsymbol{m}),
$$

which suggests that the $\hbar$-like parameter is $1 / N$ and that the classical limit corresponds to $N \rightarrow \infty$. In the following section, we set up a CS technique suited to study classical cat maps as limits of quantized cats.

\subsection{Coherent states for cat maps}

We shall construct a CS-family $\left\{\left|C_{N}(\boldsymbol{x})\right\rangle \mid \boldsymbol{x} \in \mathbb{T}^{2}\right\}$ on the 2-torus by means of the discrete Weyl group. We define

$$
\left|C_{N}(\boldsymbol{x})\right\rangle:=W_{N}(\lfloor N \boldsymbol{x}\rfloor)\left|C_{N}\right\rangle
$$

where $\lfloor N \boldsymbol{x}\rfloor=\left(\left\lfloor N x_{1}\right\rfloor,\left\lfloor N x_{2}\right\rfloor\right), 0 \leq\left\lfloor N x_{i}\right\rfloor \leq N-1$ is the largest integer smaller than $N x_{i}$ and the reference vector $\left|C_{N}\right\rangle$ is chosen to be

$$
\left|C_{N}\right\rangle=\sum_{j=0}^{N-1} C_{N}(j)|j\rangle, \quad C_{N}(j):=\frac{1}{2^{(N-1) / 2}} \sqrt{\left(\begin{array}{c}
N-1 \\
j
\end{array}\right)} .
$$

Measurability and normalization are immediate, overcompleteness comes as follows. Let $Y$ be the operator in the left hand side of Definition 3.1. If $\tau_{N}\left(Y W_{N}(\boldsymbol{n})\right)=$ $\tau_{N}\left(W_{N}(\boldsymbol{n})\right)$ for all $\boldsymbol{n}=\left(n_{1}, n_{2}\right)$ with $0 \leq n_{i} \leq N-1$, then according to (12) applied to $Y$ it follows that $Y=\mathbb{1}$. This is indeed the case as, using Eqs. (7b), (13) and $N$-periodicity,

$$
\begin{aligned}
\tau_{N}\left(Y W_{N}(\boldsymbol{n})\right)=\int_{\mathbb{T}^{2}} \mathrm{~d} \boldsymbol{x}\left\langle C_{N}(\boldsymbol{x}), W_{N}(\boldsymbol{n}) C_{N}(\boldsymbol{x})\right\rangle \\
\quad=\int_{\mathbb{T}^{2}} \mathrm{~d} \boldsymbol{x} \exp \left(\frac{2 \pi \mathrm{i}}{N} \sigma(\boldsymbol{n},\lfloor N \boldsymbol{x}\rfloor)\right)\left\langle C_{N}, W_{N}(\boldsymbol{n}) C_{N}\right\rangle
\end{aligned}
$$




$$
\begin{aligned}
& =\frac{1}{N^{2}} \sum_{\boldsymbol{p} \in(\mathbb{Z} / N \mathbb{Z})^{2}} \exp \left(\frac{2 \pi \mathrm{i}}{N} \sigma(\boldsymbol{n}, \boldsymbol{p})\right)\left\langle C_{N}, W_{N}(\boldsymbol{n}) C_{N}\right\rangle \\
& =\tau_{N}\left(W_{N}(\boldsymbol{n})\right) .
\end{aligned}
$$

In the last line we used that when $\boldsymbol{x}$ runs over $\mathrm{T}^{2},\left\lfloor N x_{i}\right\rfloor, i=1,2$ runs over the set of integers $0,1, \ldots, N-1$.

The proof of the localization property in Definition 3.1 is more technical and requires several steps: the willing reader can find it in $[27,28]$.

\section{Quantum and classical time evolutions}

One of the main issues in the semi-classical analysis is to compare if and how the quantum and classical time evolutions mimic each other when a quantization parameter goes to zero.

In the case of classically chaotic quantum systems, the situation is strikingly different from the case of classically integrable quantum systems. In the former case, classical and quantum mechanics agree on the level of coherent states only over times which scale as $-\log \hbar$.

As before, let $T$ denote the evolution on the classical phase space $\mathcal{X}$ and $U_{T}$ the unitary single step evolution on $\mathbb{C}^{N}$, the so-called Floquet operator, which represent its "quantization". We formally state the semi-classical correspondence of classical and quantum evolution using coherent states:

Condition 5.1. Dynamical localization: There exists an $\alpha>0$ such that for all choices of $\varepsilon>0$ and $d_{0}>0$ there exists an $N_{0} \in \mathbb{N}$ with the following property: if $N>N_{0}$ and $k \leq \alpha \log N$, then $N\left|\left\langle C_{N}(\boldsymbol{x}), U_{T}^{k} C_{N}(\boldsymbol{y})\right\rangle\right|^{2} \leq \varepsilon$ whenever $d\left(T^{k} \boldsymbol{x}, \boldsymbol{y}\right) \geq d_{0}$.

Remark 5.1. The condition of dynamical localization is what is expected of a good choice of coherent states, namely, on a timescale logarithmic in the inverse of the semi-classical parameter, evolving CS should stay localized around the classical trajectories. Informally, when $N \rightarrow \infty$, the quantities

$$
K_{k}(\boldsymbol{x}, \boldsymbol{y}):=\left\langle C_{N}(\boldsymbol{x}), U_{T}^{k} C_{N}(\boldsymbol{y})\right\rangle
$$

should behave as if $N\left|K_{k}(\boldsymbol{x}, \boldsymbol{y})\right|^{2} \simeq \delta\left(T^{k} \boldsymbol{x}-\boldsymbol{y}\right.$ ) (let us note that this hypothesis makes our quantization consistent with the notion of regular quantization described in Sect. $\mathrm{V}$ of [21]). The constraint $k \leq \alpha \log N$ is typical of hyperbolic classical behavior and comes heuristically as follows. The maximal localization of coherent states cannot exceed the minimal coarse-graining dictated by $1 / N$; if, while evolving, CS stayed localized forever around the classical trajectories, they would get more and more localized along the contracting direction. Since for hyperbolic systems the increase in localization is exponential with Lyapunov exponent $\log \lambda_{+}>0$, this sets the upper bound, better known as logarithmic breaking-time, and indicates that $\alpha \simeq 1 / \log \lambda_{+}$.

Proposition 5.1. Let $\left(\mathcal{M}_{N}, \Theta_{N}, \tau_{N}\right)$ be a general quantum dynamical system as defined in Sect. 3 and suppose that it satisfies Condition 5.1. Let $\|X\|_{2}:=$ 
$\sqrt{\tau_{N}\left(X^{*} X\right)}, X \in \mathcal{M}_{N}$ denote the normalized Hilbert-Schmidt norm. In the ensuing topology

$$
\lim _{k, N \rightarrow \infty, k<\alpha \log N}\left\|\Theta_{N}^{k} \circ \gamma_{N \infty}(f)-\gamma_{N \infty} \circ \Theta^{k}(f)\right\|_{2}=0 .
$$

Remark 5.2. The above proposition, whose proof can be found in [27, 28], can be seen as a modification of the so-called Egorov's property (see [36]), and gives the strength of the non-commutativity of classical and time limits when the classical system has a positive Lyapunov exponent. The same (logarithmic) scaling for the breaking-time has been found numerically in [37] also for discrete classical cat maps, converging in a suitable classical limit to continuous cat maps. Analogously, similar analysis [38] has been performed on sequences of discrete approximants of discontinuous automorphisms on the 2-torus, known as Sawtooth maps, and the logarithmic breaking-time has been recovered there, too.

We shall not prove the dynamical localization condition 5.1 for the quantum cat maps, but a direct derivation of formula (16), based on the simple expression (8) of the dynamics when acting on Weyl operators, is available in [27, 28] and reads as follows:

Proposition 5.2. Let $\left(\mathcal{M}_{N}, \Theta_{N}, \tau_{N}\right)$ be a sequence of quantum cat maps tending with $N \rightarrow \infty$ to a classical cat map with Lyapunov exponent $\log \lambda_{+}$; then

$$
\lim _{k, N \rightarrow \infty, k<\log N /\left(2 \log \lambda_{+}\right)}\left\|\Theta_{N}^{k} \circ \gamma_{N \infty}(f)-\gamma_{N \infty} \circ \Theta^{k}(f)\right\|_{2}=0,
$$

where $\|\cdot\|_{2}$ is the Hilbert-Schmidt norm of Proposition 5.1.

\section{Dynamical entropies}

Intuitively, one expects the instability proper to the presence of a positive Lyapunov exponent to correspond to some degree of unpredictability of the dynamics: classically, the metric entropy of Kolmogorov provides the link [8].

$$
\text { 6.1. Kolmogorov metric entropy }
$$

For continuous classical systems $(\mathcal{X}, T, \mu)$ such as those introduced in Sect. 2 , the construction of the dynamical entropy of Kolmogorov is based on subdividing $\mathcal{X}$ into measurable disjoint subsets $\left\{E_{\ell} \mid \ell=1,2, \cdots, D\right\}$ such that $\bigcup_{\ell} E_{\ell}=\mathcal{X}$ which form finite partitions (coarse graining) $\mathcal{E}$.

Under the dynamical maps $T: \mathcal{X} \rightarrow \mathcal{X}$, any given $\mathcal{E}$ evolves into $T^{j}(\mathcal{E})$ with atoms $T^{-j}\left(E_{\ell}\right)=\left\{\boldsymbol{x} \in \mathcal{X} \mid T^{j} \boldsymbol{x} \in E_{\ell}\right\}$; one can then form finer partitions

$$
\mathcal{E}_{[0, n-1]}:=\bigvee_{j=0}^{n-1} T^{j}(\mathcal{E})=\mathcal{E} \bigvee T(\mathcal{E}) \bigvee \cdots \bigvee T^{n-1}(\mathcal{E})
$$

whose atoms

$$
E_{i_{0} i_{1} \cdots i_{n-1}}:=\bigcap_{j=0}^{n-1} T^{-j} E_{i_{j}}=E_{i_{0}} \bigcap T^{-1}\left(E_{i_{1}}\right) \bigcap \cdots \bigcap T^{-n+1}\left(E_{i_{n-1}}\right)
$$

have volumes

$$
\mu_{i_{0} i_{1} \cdots i_{n-1}}:=\mu\left(E_{i_{0} i_{1} \cdots i_{n-1}}\right) .
$$


Definition 6.1. We shall set $\boldsymbol{i}=\left\{i_{0} i_{1} \cdots i_{n-1}\right\}$ and denote by $\Omega_{D}^{n}$ the set of $D^{n}$ $n_{-}$tuples with $i_{j}$ taking values in $\{1,2, \cdots, D\}$.

The atoms of the partitions $\mathcal{E}_{[0, n-1]}$ describe segments of trajectories up to time $n$ encoded by the atoms of $\mathcal{E}$ that are traversed at successive times; the volumes $\mu_{\boldsymbol{i}}=\mu\left(E_{\boldsymbol{i}}\right)$ corresponds to probabilities for the system to belong to the atoms $E_{i_{0}}, E_{i_{1}}, \cdots, E_{i_{n-1}}$ at successive times $0 \leq j \leq n-1$. The $n_{-}$tuples $i$ by themselves provide a description of the system in a symbolic dynamic.

The richness in diverse trajectories, that is the degree of irregularity of the motion (as seen with the accuracy of the given coarse-graining) correspond intuitively to our idea of "complexity" and can be better measured by the Shannon entropy [39]:

$$
S_{\mu}\left(\mathcal{E}_{[0, n-1]}\right):=-\sum_{i \in \Omega_{D}^{n}} \mu_{i} \log \mu_{i} .
$$

In the long run, $\mathcal{E}$ attributes to the dynamics an entropy per unit time-step

$$
h_{\mu}(T, \mathcal{E}):=\lim _{n \rightarrow \infty} \frac{1}{n} S_{\mu}\left(\mathcal{E}_{[0, n-1]}\right) .
$$

This limit is well defined [3] and the "average entropy production" $h_{\mu}(T, \mathcal{E})$ measure how predictable the dynamics is on the coarse grained scale provided by the finite partition $\mathcal{E}$. To remove the dependence on $\mathcal{E}$, the Kolmogorov-Sinai entropy $h_{\mu}^{\mathrm{KS}}(T)$ of $(\mathcal{X}, T, \mu)$ (or KS-entropy) is defined as the supremum over all finite measurable partitions $[3,39$ :

$$
h_{\mu}^{\mathrm{KS}}(T):=\sup _{\mathcal{E}} h_{\mu}(T, \mathcal{E}) .
$$

For the automorphisms of the 2-torus, we have the well-known result [3]:

Proposition 6.1. Let $\left(\mathfrak{M}_{\mu}, \Theta, \omega_{\mu}\right)$ be as in Definition 4.1, then $h_{\mu}^{\mathrm{KS}}(T)=\log \lambda_{+}$.

\subsection{Quantum dynamical entropies}

The idea behind the notion of dynamical entropy is that information can be obtained by repeatedly observing a system in the course of its time evolution. Due to the uncertainty principle, or, in other words, to non-commutativity, if observations are intended to gather information about the intrinsic dynamical properties of quantum systems, then non-commutative extensions of the KS-entropy ought first to decide whether quantum disturbances produced by observations have to be taken into account or not.

Concretely, let us consider a quantum system described by a density matrix $\rho$ acting on a Hilbert space $\mathfrak{H}$. Via the wave packet reduction postulate, generic measurement processes may be described by finite sets $\mathcal{Y}=\left\{y_{1}, y_{2}, \ldots, y_{D}\right\}$ of bounded operators $y_{j} \in \mathcal{B}(\mathfrak{H})$ such that $\sum_{j} y_{j}^{*} y_{j}=\mathbb{1}$. These sets are called partitions of unity (p.u., for the sake of shortness) and describe the change in the state of the system caused by the corresponding measurement process

$$
\rho \mapsto \Gamma_{\mathcal{Y}}^{*}(\rho):=\sum_{j} y_{j} \rho y_{j}^{*}
$$

It looks rather natural to rely on partitions of unity to describe the process of 
collecting information through repeated observations of an evolving quantum system [18]. Yet, most of these measurements interfere with the quantum evolution, possibly acting as a source of unwanted extrinsic randomness. Nevertheless, the effect is typically quantal and rarely avoidable. Quite interestingly, as we shall see later, pursuing these ideas leads to quantum stochastic processes with a quantum dynamical entropy of their own, the ALF-entropy, that is also useful in a classical context.

An alternative approach [17] leads to the dynamical entropy of Connes et al. [17] (CNT-entropy). This approach lacks the operational appeal of the ALF-construction, but is intimately connected with the intrinsic relaxation properties of quantum systems $[17,40]$ and possibly useful in the rapidly growing field of quantum communication. The CNT-entropy is based on decomposing quantum states rather than on reducing them as in (21). Explicitly, if the state $\rho$ is not a one-dimensional projection, any partition of unity $\mathcal{Y}$ yields a decomposition

$$
\rho=\sum_{j} \operatorname{Tr}\left(\rho y_{j}^{*} y_{j}\right) \frac{\sqrt{\rho} y_{j}^{*} y_{j} \sqrt{\rho}}{\operatorname{Tr}\left(\rho y_{j}^{*} y_{j}\right)} .
$$

When $\Gamma_{\mathcal{Y}}^{*}(\rho)=\rho$, reductions also provide decompositions, but not in general.

A different kind of wave packet reduction is the starting point for constructing the coherent states entropy [21, 41] (in the following CS-entropy, for short), in fact based on coherent states $\left|C_{N} \boldsymbol{x}\right\rangle$ as the ones introduced by Definition 3.1.

The map

$$
\mathcal{I}(E)(\rho):=N \int_{E}\left|C_{N}(\boldsymbol{x})\right\rangle\left\langle C_{N}(\boldsymbol{x})|\rho| C_{N}(\boldsymbol{x})\right\rangle\left\langle C_{N}(\boldsymbol{x})\right| \mu(\mathrm{d} \boldsymbol{x}),
$$

for a measurable subset $E \subset \mathcal{X}$ and an operator $\rho$, is called an instrument: it describes the change in the state $\rho$ of the system caused by an $E$-dependent measurement process (compare with (21)), actually a double approximate measurement in the phase space. Repeated measurement, taken stroboscopically during the dynamical evolution and performed with different instrument $\mathcal{I}\left(E_{i_{j}}\right)$ labeled by different elements $E_{i_{j}}$ of a partition $\mathcal{E}$, map the input state $\rho$ into many possible outputs $\left\{\rho_{\boldsymbol{i}} \mid \boldsymbol{i} \in \Omega_{D}^{n}\right\}$, which in turn can be mapped into many positive numbers $\left\{\mathbb{R}^{+} \ni \omega_{\boldsymbol{i}}:=\omega \rho_{\boldsymbol{i}} \mid \boldsymbol{i} \in \Omega_{D}^{n}\right\}$ summing up to one. Now we have once more the correspondence between strings $\boldsymbol{i} \in \Omega_{D}^{n}$ and probability $\omega_{\boldsymbol{i}}$, in other words we end up with a probability space and a similar reasoning leading us in Sect. 6.1 to the KS invariant, can now be used for constructing the CS-entropy.

\subsection{ALF-entropy}

The idea underlying the ALF-entropy is that the evolution of a quantum dynamical system can be modeled by repeated measurements at successive equally spaced times, the measurements corresponding to p.u. as in Eq. (21).

Such a construction associates a quantum dynamical system with a symbolic dynamics corresponding to the right-shift along a quantum spin half-chain [42].

Generic p.u. $\mathcal{Y}=\left\{y_{1}, y_{2}, \ldots, y_{D}\right\}$ need not preserve the state, but disturbances are kept under control by suitably selecting the subalgebra of observables 
$\mathfrak{M}_{0} \ni y_{j}$. The construction of the ALF-entropy for a quantum dynamical system $(\mathfrak{M}, \Theta, \omega)$ can be resumed as follows:

- One selects a $\Theta$-invariant subalgebra $\mathfrak{M}_{0} \subseteq \mathfrak{M}$ and a p.u. $\mathcal{Y}=\left\{y_{1}, \ldots, y_{D}\right\}$ of finite size $D$ with $y_{j} \in \mathfrak{M}_{0}$. After $j$ time steps $\mathcal{Y}$ will have evolved into another p.u. from $\mathfrak{M}_{0}: \Theta^{j}(\mathcal{Y}):=\left\{\Theta^{j}\left(y_{1}\right), \Theta^{j}\left(y_{2}\right), \ldots, \Theta^{j}\left(y_{D}\right)\right\} \subset \mathfrak{M}_{0}$.

- Every p.u. $\mathcal{Y}$ of size $D$ gives rise to a $D$-dimensional density matrix

$$
\rho[\mathcal{Y}]_{i, j}:=\omega\left(y_{j}^{*} y_{i}\right),
$$

with von Neumann entropy $H_{\omega}[\mathcal{Y}]:=S(\rho[\mathcal{Y}])=-\operatorname{Tr}(\rho[\mathcal{Y}] \log \rho[\mathcal{Y}])$.

- Given two partitions of unit $\mathcal{Y}=\left\{y_{1}, y_{2}, \ldots, y_{D}\right\}, \mathcal{Z}=\left\{z_{1}, z_{2}, \ldots, z_{B}\right\}$, of size $D$, respectively $B$, one gets a finer partition of unit of size $B D$ as the set

$$
\mathcal{Y} \circ \mathcal{Z}:=\left\{y_{1} z_{1}, \ldots, y_{1} z_{B} ; y_{2} z_{1}, \ldots, y_{2} z_{B} ; \ldots ; y_{D} z_{1}, \ldots, y_{D} z_{B}\right\}
$$

- Given a size $D$ p.u. $\mathcal{Y}$ and the ordered time refinements

$$
\mathcal{Y}^{[0, n-1]}:=\Theta^{n-1}(\mathcal{Y}) \circ \Theta^{n-2}(\mathcal{Y}) \circ \cdots \circ \mathcal{Y},
$$

the $D^{n} \times D^{n}$ density matrices $\rho_{\mathcal{Y}}^{[0, n-1]}:=\rho\left[\mathcal{Y}^{[0, n-1]}\right]$ define states on the $n$-fold tensor product $\mathcal{M}_{D}^{\otimes n}$ of $D$-dimensional matrix algebras $\mathcal{M}_{D}$.

- Given a p.u. $\mathcal{Y}$ of size $D$, let $\Phi_{\mathcal{Y}}: \mathcal{M}_{D} \otimes \mathfrak{M} \mapsto \mathfrak{M}$ and $e_{M}: \mathfrak{M} \mapsto \mathfrak{M}$, with $M \in \mathcal{M}_{D}$, be linear maps defined by

$$
\Phi_{\mathcal{Y}}(M \otimes x):=\sum_{i, j} y_{i}^{*} x y_{j} M_{i j} \quad \text { and } \quad e_{M}(x):=\sum_{i, j} y_{i}^{*} \Theta(x) y_{j} M_{i j}
$$

$\Phi_{\mathcal{Y}}$ is a completely positive unital map, while $e_{\mathbb{1}}(\mathbb{1})=\mathbb{1}$. One readily computes

$$
\omega\left(e_{M_{0}} \circ e_{M_{1}} \cdots \circ e_{M_{n-1}}(\mathbb{1})\right)=\operatorname{Tr}\left(\rho_{\mathcal{Y}}^{[0, n-1]} M_{0} \otimes M_{1} \cdots \otimes M_{n-1}\right) .
$$

The states $\rho_{\mathcal{Y}}^{[0, n-1]}$ are compatible

$$
\rho_{\mathcal{Y}}^{[0, n-1]}\left\lceil\mathcal{M}_{D}^{[0, n-2]}=\rho_{\mathcal{Y}}^{[0, n-2]}, \quad \text { where } \quad \mathcal{M}_{D}^{[0, n-2]}:=\bigotimes_{\ell=0}^{n-2}\left(\mathcal{M}_{D}\right)_{\ell}\right.
$$

and define a global state $\rho \mathcal{y}$ on the quantum spin chain $\mathcal{M}_{D}^{\infty}:=\otimes_{\ell=0}^{\infty}\left(\mathcal{M}_{D}\right)_{\ell}$.

Thus it is possible to associate with the quantum dynamical system $(\mathfrak{M}, \Theta, \omega)$ a symbolic dynamics which amounts to the right-shift $\sigma:\left(\mathcal{M}_{D}\right)_{\ell} \mapsto$ $\left(\mathcal{M}_{D}\right)_{\ell+1}$ along the quantum spin half-chain.

Non-commutativity becomes evident when we check whether $\rho_{\mathcal{Y}}$ is shift-invariant. This requires $\omega\left(\sum_{\ell} y_{\ell}^{*} x y_{\ell}\right)=\omega(x)$ for all $x \in \mathcal{M}$. Let us note that this is the case in which $\rho \mapsto \Gamma_{\mathcal{Y}}^{*}(\rho)=\rho$ (see Eq. (21)).

Definition 6.2. The ALF-entropy of a quantum dynamical system $(\mathfrak{M}, \Theta, \omega)$ is

$$
h_{\omega, \mathcal{M}}^{\mathrm{ALF}}(\Theta):=\sup _{\mathcal{Y} \subset \mathcal{M}_{0}} h_{\omega}^{\mathrm{ALF}}(\Theta, \mathcal{Y})
$$

where 


$$
h_{\omega}^{\mathrm{ALF}}(\Theta, \mathcal{Y}):=\lim _{n} \sup \frac{1}{n} H_{\omega}\left[\mathcal{Y}^{[0, n-1]}\right] .
$$

Like the metric entropy of a partition $\mathcal{E}$, also the ALF-entropy of a partition of unit $\mathcal{Y}$ can be physically interpreted as an asymptotic entropy production relative to a specific coarse-graining.

\subsection{Comparison of dynamical entropies}

In this section we outline some of the main common features of many different dynamical entropies, taking the ALF as a reference example, because of its conceptual simplicity. Here, we just sketch such features, emphasizing those parts that are important to the study of the classical limit of quantum dynamical entropies (QDE).

The first thing to notice is that any QDE must coincide with the KS-entropy when $\mathfrak{M}=\mathfrak{M}_{\mu}$ is the Abelian von Neumann algebra $\mathfrak{L}_{\mu}^{\infty}(\mathcal{X})$ and $(\mathfrak{M}, \Theta, \omega)$ represents a classical dynamical system.

The next observation is that when, as for the quantized hyperbolic automorphisms of the torus considered in this paper, $\mathfrak{M}$ is a finite-dimensional algebra, both the CNT- and the ALF-entropy are zero, see $[17,18]$. Consequently, if we decide to take the strict positivity of ALF- or CNT-entropy as a signature of quantum chaos, quantized hyperbolic automorphisms of the torus cannot be called chaotic.

However, the latter observation is not as general as the former. There exist many alternative definitions (different from ALF and CNT), and some of them need not to be equal to zero for all quantum systems defined on a finite-dimensional Hilbert space: an interesting example is represented by the CS-entropy introduced in $[21]$.

From the previous considerations, it is clear that the main field of application of the CNT- and ALF-entropies are infinite quantum systems, where the differences between the two come to the fore [42].

The complete proofs of the above facts can be found in [17] for the CNT-, in $[18,43]$ for the ALF-, and in [21] for the CS-entropy. Here we just state more rigorously the above observations, in the case of the ALF-entropy, in the two subsequent Proposition 6.2 and Proposition 6.3.

Proposition 6.2. Let $\left(\mathfrak{M}_{\mu}, \Theta, \omega_{\mu}\right)$ represent a classical dynamical system. Then, with the notations of the previous sections

$$
h_{\left(\omega_{\mu}, \mathfrak{M}_{\mu}\right)}^{\mathrm{ALF}}(\Theta)=h_{\mu}^{\mathrm{KS}}(T) .
$$

In the particular case of the hyperbolic automorphisms of the torus, we may restrict our attention to p.u. whose elements belong to the $*$-algebra $\mathcal{D}_{\mu}$ of complex functions $f$ on $\mathrm{T}^{2}$ such that the support of $\hat{f}$ is bounded

$$
h_{\mu}^{\mathrm{KS}}(T)=h_{\left(\omega_{\mu}, \mathfrak{M}_{\mu}\right)}^{\mathrm{ALF}}(\Theta)=h_{\left(\omega_{\mu}, \mathcal{D}_{\mu}\right)}^{\mathrm{ALF}}(\Theta) .
$$

Remarkably, the computation of the classical KS-entropy via the quantum mechanical ALF-entropy yields a proof of Proposition 6.1 that is much simpler than the standard ones $[12,13]$. 
Proposition 6.3. Let $(\mathfrak{M}, \Theta, \omega)$ be a quantum dynamical system with $\mathfrak{M}$, a finite dimensional $C^{*}$-algebra, then

$$
h_{(\omega, \mathfrak{M})}^{\mathrm{ALF}}(\Theta)=0
$$

\section{Classical limit of quantum dynamical entropies}

Proposition 6.3 confirms the intuition that finite-dimensional, discrete time, quantum dynamical systems, however complicated the distribution of their quasienergies might be, cannot produce enough information over large times to generate a non-vanishing entropy per unit time. This is due to the fact that, despite the presence of almost random features over finite intervals, the time evolution cannot bear random signatures if watched long enough, because almost periodicity would always prevail asymptotically.

However, this does not mean that the dynamics may not be able to show a significant entropy rate over finite interval of times, these being typical of the underlying dynamics. Here we show that underlying classical chaos plus Hilbert space finiteness make a characteristic logarithmic timescale emerge over which these systems can be called chaotic. This is precisely the content of the next Theorem 7.1, whose proof can be found in [27, 28].

Theorem 7.1. Let $(\mathcal{X}, T, \mu)$ be a classical dynamical system which is the classical limit of a sequence of finite-dimensional quantum dynamical systems $\left(\mathcal{M}_{N}, \Theta_{N}, \tau_{N}\right)$. We also assume that the dynamical localization condition 5.1 holds. If

1. $\mathcal{E}=\left\{E_{1}, E_{2}, \ldots, E_{D-1}\right\}$ is a finite measurable partition of $\mathcal{X}$,

2. $\mathcal{Y}_{N}=\left\{y_{1}, y_{2}, \ldots, y_{D}\right\}$ is a bistochastic partition of unity, which is the quantization of the previous partition, namely $y_{i}=\gamma_{N \infty}\left(\chi_{E_{i}}\right)$ for $i=1,2, \ldots, D-1$ and $y_{D}:=\sqrt{\mathbb{1}-\sum_{i=0}^{D-1} y_{i}^{*} y_{i}}$,

then there exists an $\alpha$ such that

$$
\lim _{k, N \rightarrow \infty, k \leq \alpha \log N} \frac{1}{k}\left|H\left[\mathcal{Y}_{N}^{(k)}\right]-S_{\mu}\left(\mathcal{E}^{(k)}\right)\right|=0 .
$$

A similar phenomenon has been proved both for the CNT-entropy [27, 28] and for the CS-entropy [44], although in this case a different kind of dynamical system has been studied. Nevertheless, the proof of convergence of CS-entropy to the KS invariant only make use of dynamical localization condition 5.1 so that, after an appropriate substitution of similar terms, Theorem 7.1 can be extended to both CNT- and CS-entropies.

The dynamical localization condition 5.1 has been extensively used in all the proofs mentioned in this section, and the results here presented strongly do depend on it. Once the framework in which sequences of quantum approximants approach their classical limit has been settled, by an appropriate Egorov convergence, like the one in Propositions 5.1 and 5.2, we still let room for bizarre behaviors in 
the entropy production. Condition 5.1 removes such a freedom and extend the convergence from observables to dynamical entropies.

\section{Conclusions and outlook}

We have reviewed how quantum dynamical entropies reproduce the Kolmogorov-Sinai invariant in quantum systems too, provided that we observe a strongly chaotic system on a very short logarithmic timescale. However, due to the discreteness of the spectrum of the quantizations, we know that saturation phenomena will appear. It would be interesting to study the scaling behavior of the quantum dynamical entropies in the intermediate region between the logarithmic breaking time and the Heisenberg time. This will, however, require quite different techniques than the coherent states approach, indeed the Ehrenfest time, whose scaling is the same as the breaking time here described, set the upper bound of semi-classical technology.

\section{Acknowledgments}

It is a pleasure to thank F. Benatti and K. Życzkowski for stimulating discussions. The author acknowledges financial support provided by the EU Marie Curie Host Fellowships for Transfer of Knowledge Project COCOS (contract number MTKD-CT-2004-517186) and the SFB/Transregio-12 project financed by DFG.

\section{References}

[1] R. Devaney, An Introduction to Chaotic Dynamical Systems, Addison-Wesley, Reading, MA 1989.

[2] S. Wiggins, Dynamical Systems and Chaos, Springer-Verlag, New York 1990.

[3] A. Katok, B. Hasselblatt, Introduction to the Modern Theory of Dynamical Systems, Encyclopedia of Mathematics and its Applications, Cambridge University Press, Cambridge 1999.

[4] H.G. Schuster, Deterministic Chaos, 3rd ed., VCH, Weinheim 1995.

[5] Chaos and Quantum Physics, Eds. M.-J. Giannoni, A. Voros, J. Zinn-Justin, Vol. 1989, Les Houches Session LII of Les Houches Summer School of Theoretical Physics, North-Holland, Amsterdam 1991.

[6] G. Casati, B. Chirikov, Quantum Chaos. Between Order and Disorder, Cambridge University Press, Cambridge 1995.

[7] G.M. Zaslavsky, Chaos in Dynamic Systems, Harwood Academic Publ., Chur 1985.

[8] J. Ford, M. Ilg, Phys. Rev. A 45, 6165 (1992).

[9] G.M. Zaslawsky, in: Quantum Chaos, Eds. H.A. Cerdeira, R. Ramaswamy, M.C. Gutzwiller, G. Casati, World Scientific, Singapore 1991, p. 32.

[10] J. Ford, G. Mantica, G.H. Ristow, Physica D 50, 493 (1991).

[11] J. Ford, G. Mantica, Am. J. Phys. 60, 1086 (1992).

[12] V.I. Arnold, A. Avez, Ergodic Problems of Classical Mechanics, Benjamin, New York 1968. 
[13] P. Walters, An Introduction to Ergodic Theory, Vol. 79 of Graduate Text in Mathematics, Springer-Verlag, Berlin 1982.

[14] M.V. Berry, N.L. Balazs, M. Tabor, A. Voros, Ann. Phys. 122, 26 (1979).

[15] M. Degli Esposti, Ann. Inst. Henri Poincaré 58, 323 (1993).

[16] R. Mañé, Ergodic Theory and Differentiable Dynamics, Springer-Verlag, Berlin 1987.

[17] A. Connes, H. Narnhofer, W. Thirring, Commun. Math. Phys. 112, 691 (1987).

[18] R. Alicki, M. Fannes, Lett. Math. Phys. 32, 75 (1994).

[19] D. Voiculescu, Commun. Math. Phys. 144, 443 (1992).

[20] L. Accardi, M. Ohya, N. Watanabe, Open Sys. Information Dyn. 4, 71 (1997).

[21] W. Słomczyński, K. Życzkowski, J. Math. Phys. 35, 5674 (1994).

[22] R. Alicki, D. Makowiec, W. Miklaszewski, Phys. Rev. Lett. 77, 838 (1996).

[23] M. Pogorzelska, R. Alicki, J. Phys. A, Math. Theor. 40, 3379 (2007).

[24] R. Alicki, M. Fannes, Quantum Dynamical Systems, Oxford University Press, Oxford 2001.

[25] R.F. Werner, preprint quant-ph/9504016, 1995.

[26] W. Rudin, Real and Complex Analysis, 3rd ed., McGraw-Hill, New York 1987.

[27] F. Benatti, V. Cappellini, M. De Cock, M. Fannes, D. Vanpeteghem, Rev. Math. Phys. 15, 847 (2003).

[28] V. Cappellini, Ph.D. thesis, University of Trieste 2004, math-ph/0403035.

[29] I.C. Percival, F. Vivaldi, Physica D 27, 373 (1987).

[30] F. Benatti, H. Narnhofer, G.L. Sewell, Lett. Math. Phys. 21, 157 (1991).

[31] S. De Bièvre, in: Quantization, Coherent States and Poisson Structures, Proc. XIVth Workshop on Geometrical Methods in Physics, Bialowieza (Poland) 1995, Eds. A. Strassburger, S. Twareque Ali, J.-P. Antoine, J.-P. Gazeau, A. Odzijewicz, Polish Scientific Publisher PWN, Warsaw 1998, preprint mp_arc 96-191.

[32] M.V. Berry, J.H. Hannay, Physica D 1, 267 (1980).

[33] J.P. Keating, Nonlinearity 4, 309 (1991).

[34] J.P. Keating, F. Mezzadri, ibid. 13, 747 (2000).

[35] F. Mezzadri, ibid. 15, 905 (2002).

[36] J. Marklof, Z. Rudnick, GAFA, Geom. Funct. Anal. 10, 1554 (2000).

[37] F. Benatti, V. Cappellini, F. Zertuche, J. Phys. A, Math. Gen. 37, 105 (2004).

[38] F. Benatti, V. Cappellini, J. Math. Phys. 46, 062702 (2005).

[39] V.M. Alekseev, M.V. Yakobson, Phys. Rep. 75, 287 (1981).

[40] H. Narnhofer, J. Math. Phys. 33, 1502 (1992).

[41] W.S. Słomczyński, K. Życzkowski, Phys. Rev. Lett. 80, 1880 (1998).

[41] P. Tuyls, M.Sc. thesis, K.U.-Leuven, 1997.

[42] R. Alicki, H. Narnhofer, Lett. Math. Phys. 33, 241 (1995).

[43] R. Alicki, J. Andries, M. Fannes, P. Tuyls, Rev. Math. Phys. 8, 167 (1996).

[44] V. Cappellini, J. Phys. A, Math. Gen. 38, 6893 (2005). 